\title{
Rayleigh Distribution and Its Generalizations
}

\author{
Petr Beckmann ${ }^{1}$ \\ Department of Electrical Engineering, University of Colorado, Boulder, Colo.
}

(Received December 7, 1963)

\begin{abstract}
The Rayleigh distribution is the distribution of the sum of a large number of coplanar (or time) vectors with random amplitudes and uniformly distributed phases. As such, it is the limiting case of distributions associated with more general vector sums that arise in practical problems. Such cases are the following: (a) The phase distributions of the vector terms are not uniform, e.g., in the case of scattering from rough surfaces; (b) One or more vector terms predominate, their mean square value not being negligible compared to the mean square value of the sum, e.g., in the case of signals propagated in cities, meteor-scatter, and atmospheric noise; (e) The number of vector terms is small, e.g., in radar returns from several close targets; (d) The number of vector terms is itself random, e.g., in atmospheric turbulence, meteor-scatter and atmospheric noise. The resulting distributions for these cases and their deviations from the Rayleigh distribution will be considered.
\end{abstract}

\section{Introduction}

In many problems arising in radio wave propagation the resultant field is formed by the superposition or interference of a number of elementary waves:

$$
E e^{i \theta}=\sum_{j=1}^{n} E_{j} e^{i \phi_{j}}
$$

where the $E_{j}$ and the $\phi_{j}$ and even $n$ may be random, and the distributions of the individual terms of the sum need not be identical. One is then faced with the problem of determining the distribution of $E$ (and sometimes of $\theta$ ) if the distributions of the $E_{j}$, the $\phi_{j}$ and $n$ are known. It will be assumed that the terms of the sum (1) are mutually independent. The sum (1) may also be regarded as the sum of coplanar vectors.

In its most elementary form, when $n$ is a large constant, the $E_{j}$ are all equal to the same constant, and the $\phi_{j}$ are all uniformly distributed from 0 to $2 \pi$, the problem was solved by Rayleigh [1896] and leads to the well-known Rayleigh distribution

$$
p(E)=\frac{2 E}{s} e^{-E^{2} / s},
$$

where $s=\left\langle E^{2}\right\rangle=n E_{j}^{2}$ and the brackets $\langle\quad\rangle$ denote the mean value; or

1 On leave of absence from the Institute of Radio Engineering and Electronics, Czechoslovak Academy of Sciences, Prague.

$$
P\left(E / E_{\mathrm{RMS}}>R\right)=e^{-R^{2}} ; E_{\mathrm{RMS}}=\sqrt{s} \cdot \ldots
$$

The present paper considers (1) under more general conditions. In principle the problem can always be solved by resolving (1) into its rectangular components (i.e., real and imaginary parts) $x$ and $y$,

$$
\left.\begin{array}{l}
x=E \cos \theta=\sum_{j=1}^{n} E_{j} \cos \phi_{j}=\sum_{j=1}^{n} x_{j} \\
y=E \sin \theta=\ldots
\end{array}\right\}
$$

finding the joint probability density $W(x, y)$, and retransforming to polar coordinates through

$$
\begin{aligned}
& p(E)=E \int_{0}^{2 \pi} W(E \cos \theta, E \sin \theta) d \theta \\
& p_{\theta}(\theta)=\int_{0}^{\infty} E W(E \cos \theta, E \sin \theta) d E .
\end{aligned}
$$

The various cases that mav arise are conveniently classified according to whether or not the sums (4) satisfy the Central Limit Theorem, i.e., whether or not $x$ and $y$ are normally distributed as $n \rightarrow \infty$. Each of these two cases again includes several further possibilities.

Let $D(x)$ denote the variance of $x$; then the distribution of $x$ will tend to a normal distribution as $n \rightarrow \infty$ provided that the $x_{j}$ are mutually independent and that

$$
\lim _{n \rightarrow \infty} \frac{D\left(x_{j}\right)}{D(x)}=0 \text { for all } j
$$


with a similar statement for $y$. (This statement usually suffices for engineering purposes; for a more rigorous enunciation of the Central Limit Theorem and Lindeberg conditions ef. Gnedenko and Kolmogorov [1954].) Condition (6) essentially means that none of the terms $x_{j}$ must predominate in the resulting sum $x$. However, if the $x_{j}$ are themselves normally distributed, then $x$ will, of course, be also normally distributed even if (6) does not hold.

In most (but not all) applications the $\phi_{j}$ are distributed uniformly between 0 and $2 \pi$ or in an equivalent manner; i.e., the phase distribution $w_{\phi}(\phi)$ is such that

$$
\sum_{k=-\infty}^{\infty} w_{\phi}(\phi+2 k \pi)=\frac{1}{2 \pi}+\epsilon(\phi) ; \quad|\epsilon(\phi)|<<\frac{1}{2 \pi} .
$$

A vector with such a Uniformly Distributed Phase will be called a UDP vector. The sum of UDP vectors is obviously itself a UDP vector. If the terms in (1) are UDP vectors, then

$$
\begin{aligned}
\left\langle E^{2}\right\rangle=\left\langle\sum_{j} E_{j} e^{i \phi_{j}}\right. & \left.\sum_{k} E_{k} e^{-i \phi_{k}}\right\rangle \\
= & \sum_{j}\left\langle E_{j}^{2}\right\rangle+\sum_{j \neq k}\left\langle E_{j} E_{k}\right\rangle\left\langle e^{i\left(\phi_{j}-\phi_{k}\right)}\right\rangle .
\end{aligned}
$$

But for $j \neq k$,

$$
\left\langle e^{i\left(\phi_{j}-\phi_{k}\right)}\right\rangle=\frac{1}{4 \pi^{2}} \int_{0}^{2 \pi} \int_{0}^{2 \pi} e^{i\left(\phi_{j}-\phi_{k}\right)} d \phi_{j} d \phi_{k}=0 .
$$

Substituting (9) in (8) we find the important relation

$$
\left\langle E^{2}\right\rangle=\sum_{j=1}^{n}\left\langle E_{j}\right\rangle^{2}
$$

valid for UDP vectors regardless of the value of $n$ or the distributions of the $E_{j}$ (possibly all different).

\section{Rayleigh Distribution}

If the terms in (1) are UDP vectors, then from (4)

$$
\langle x\rangle=\langle y\rangle=0
$$

$D\left(x_{j}\right)=D\left(y_{j}\right)=\frac{1}{2 \pi} \int_{0}^{\infty} \int_{0}^{2 \pi} E_{j}^{2} \cos ^{2} \phi_{j} d E_{j} d \phi_{j}=\frac{1}{2}\left\langle E_{j}^{2}\right\rangle$.

Then using (10), condition (6) becomes

$$
\lim _{n \rightarrow \infty} \frac{\left\langle E_{j}^{2}\right\rangle}{\sum_{j=1}^{n}\left\langle E_{j}^{2}\right\rangle}=0 \text { for all } j
$$

If (13) is satisfied and $n$ is large, $x$ and $y$ can be approximated by a normal distribution with mean value zero and the same variance. The integration (5) then leads to the Rayleigh distribution (2) with

$$
s=\sum_{j=1}^{n}\left\langle E_{j}^{2}\right\rangle
$$

Thus a Rayleigh vector is a UDP vector whose $x$ and $y$ components are distributed normally with $\langle x\rangle=\langle y\rangle=0$ and $D(x)=D(y)=s / 2$. From this it follows that the sum of any number of Rayleigh vectors is itself a Rayleigh vector.

A Rayleigh distribution will thus be found whenever the resultant field is composed of a large number of UDP vectors and (13) is satisfied.

\section{Nonuniform Phase Distributions}

In a number of applications the phases $\phi_{j}$ in (1) are not distributed uniformly as in (7), but fluctuate about some privileged value. This will occur in scattering from rough surfaces (e.g., rough layers in the atmosphere) for small roughness or small grazing angles. Since the terms in (1) are now not UDP vectors, (11) and (12) will not hold. However, if (6) holds, $x$ and $y$ will still be normally distributed. If the phase distributions are symmetrical about zero, then $\langle y\rangle=0$. By the usual rules of probability theory one then finds the quantities

$$
\alpha=\langle x\rangle, s_{1}=D(x), s_{2}=D(y)
$$

and the integration (5) yields

$$
\begin{aligned}
& p(E)=\frac{E}{\sqrt{s_{1} s_{2}}} \exp \left[-\frac{\alpha^{2}}{2 s_{1}}-\frac{s_{1}+s_{2}}{4 s_{1} s_{2}} E^{2}\right] \\
& \times \sum_{m=0}^{\infty}(-)^{m} \epsilon_{m} I_{m}\left(\frac{s_{2}-s_{1}}{4 s_{2} s_{1}} r^{2}\right) I_{2 m}\left(\frac{\alpha}{s_{1}} r\right)
\end{aligned}
$$

where $I_{m}$ is the modified Bessel function of order $m$ and $\epsilon_{0}=1, \epsilon_{m}=2$ for $m \neq 0$. Details of the procedure and curves of (15) will be found in [Beckmann, 1962a].

The general distribution (15) simplifies in certain special cases. If $\alpha=0$, but $s_{1} \neq s$, then (15) reduces to

$$
p(E)=\frac{E}{\sqrt{s_{1} s_{2}}} \exp \left[-\frac{s_{1}+s_{2}}{4 s_{1} s_{2}} E^{2}\right] I_{0}\left(\frac{s_{2}-s_{1}}{4 s_{2} s_{1}} r^{2}\right),
$$

a distribution derived directly by Hoyt [1947].

On the other hand, if $s_{1}=s_{2}=\frac{1}{2} s$, but $\alpha \neq 0$, then (15) reduces to the Nakagami-Rice distribution

$$
p(E)=\frac{2 E}{s} \exp \left[-\frac{\alpha^{2}+E^{2}}{s}\right] I_{0}\left(\frac{2 \alpha E}{s}\right)
$$

a distribution derived by Rice [1944 and 1945] ${ }^{2}$ and further analyzed, e.g., by Norton et al. [1955], and Zuhrt [1957]. The distribution (17) is obtained when a constant vector $\left(E_{1}=\alpha, \phi_{1}=0\right)$ is added to a

2 The distribution was originally derived by Nakagami in 1940. A summary and bibliography of the work on this and related topics by Nakagami and other Japanese scientists will be found in [Nakagami, 1960]. 
Rayleigh vector, for the $x$ and $y$ components of the sum will then obviously be distributed normally with $\langle x\rangle=\alpha,\langle y\rangle=0, \quad D(x)=D(y)=s / 2 \quad$ (where $s$ is the mean square value of the Rayleigh vector) just as assumed in deriving (17). It should be noted that (17) will equally well hold for $E_{1} \exp \left(i \phi_{1}\right)$ a UDP vector with constant amplitude $E_{1}=\alpha$; this may be shown by measuring the phases from $\phi_{1}$ as a reference phase: the distributions of $\phi_{j}^{\prime}=\phi_{j}-\phi_{1}$ will for $j \neq 1$ remain uniform as before, whereas $\phi_{1}^{\prime}=0$, thus reducing to the same conditions under which (17) was derived.

The Rayleigh distribution, as may easily be verified, is the limiting distribution of (15), and of its special cases (16) and (17), for $\alpha=0 ; s_{1}=s_{2}=s / 2$.

If the Central Limit Theorem may be applied to (4), so that $x$ and $y$ will be distributed normally, then in the most general case (corresponding to asymmetrical phase distributions) we have four parameters:

$$
\langle x\rangle=\alpha ;\langle y\rangle=\beta ; D(x)=s_{1} ; D(y)=s_{2} .
$$

The integration (5) then leads to

$$
\begin{aligned}
& p(E)=\frac{r e^{-Z}}{\sqrt{s_{1} s_{2}}} \sum_{m=0}^{\infty}(-)^{m} \epsilon_{m} I_{m}(P) I_{2 m}\left(\sqrt{Q^{2}+R^{2}}\right) \\
& \cos \left[2 m\left(\arctan \frac{R}{Q}\right)\right]
\end{aligned}
$$

where

$$
Z=\frac{s_{1}+s_{2}}{4 s_{1} s_{2}} r^{2}+\frac{\alpha^{2}}{2 s_{1}}+\frac{\beta^{2}}{2 s_{2}} ; P=\frac{s_{2}-s_{1}}{4 s_{1} s_{2}} r^{2} ; Q=\frac{r \alpha}{s_{1}} ; R=\frac{r \beta}{s_{2}} .
$$

The distribution (19) was found by Nakagami [1960]; it is the most general distribution for the case when the Central Limit Theorem is applicable to (4); for $\beta=0$ and hence $R=0$, it reduces to (15). The Rayleigh distribution is again obtained from (19) for $\alpha=\beta=0 ; s_{1}=s_{2}=s / 2$.

If the Central Limit Theorem is not applicable to (4), this may be for one of the following reasons: (a) condition (6) is not satisfied (this will be considered in secs. 4 and 5); (b) the number of terms $n$ in (1) is not large (sec. 6) or random (sec. 7).

\section{Dominant Terms}

For UDP vectors, which we shall henceforth assume, (6) reduces to (13). If the number of interfering waves $n$ is large, but finite, we may replace (13) by

$$
\left\langle E_{j}^{2}\right\rangle\left\langle<\sum_{j=1}^{n}\left\langle E_{j}^{2}\right\rangle \text { for any } j .\right.
$$

Now if one (or more) of the interfering waves is powerful, so that its power is not negligible when compared to the total power, (21) will be violated for one (or more) $j$. If more than one of such waves are present, we may sum them by standard methods (convolutions, characteristic functions) and regard this partial sum as one wave. Since assumption (21) will hold for all other $j$, the remaining terms will add up to a Rayleigh vector, so that the problem reduces to finding the distribution of the sum of a UDP vector with random amplitude $E_{1}$ and a Rayleigh vector.

This problem may be solved directly from first principles by (4) to (5) or more quickly by randomizing $\alpha=E_{1}$ in (17) and using the theorem of total probability: if the density of $\alpha$ is $w(\alpha)$, then (17) gives the density $p(E \mid \alpha)$, so that the required total probability density is

$$
p(E)=\frac{2 E}{s} \int_{0}^{\infty} w(\alpha) \exp \left(-\frac{\alpha^{2}+E^{2}}{s}\right) I_{0}\left(\frac{2 \alpha E}{s}\right) d \alpha .
$$

It may be verified from $(22)$ that $p(E)$ will approach a Rayleigh distribution for $\left\langle\alpha^{2}\right\rangle\langle<s$, as was to be expected. The complement of the distribution function of (22) is

$$
P(E>R)=\int_{R}^{\infty} p(E) d E=\int_{0}^{\infty} w(\alpha) f(R, \alpha) d \alpha
$$

where the order of integration has been reversed with

$$
f(R, \alpha)=\frac{2}{s} \int_{R}^{\infty} E \exp \left[-\frac{\alpha^{2}+E^{2}}{s}\right] I_{0}\left(\frac{2 \alpha E}{s}\right) d E .
$$

Now if $R$ is large $(R>>s / \alpha)$, the Bessel function in (24) may be replaced by its asymptotic expression: a saddle-point integration then leads to

$$
f(R, \alpha)=\frac{1}{2}\left[1-\operatorname{erf}\left(\frac{R-\alpha}{\sqrt{s}}\right)\right] .
$$

Now (25) changes its value from $<0.01$ to $>0.99$ near the point $\alpha=R$ within an interval $\Delta \alpha=2.3 \sqrt{s} R$, tending to zero below and to unity above that interval; for $R>\sqrt{s}$ we may therefore well approximate (25) by

$$
f(R, \alpha) \approx\left\{\begin{array}{l}
0 \text { for } \alpha<R \\
1 \text { for } \alpha>R .
\end{array}\right.
$$

Substituting this value in (23) we find

$$
P(E>R) \approx \int_{R}^{\infty} w(\alpha) d \alpha=P(\alpha>R) \quad(\text { for } R>>\sqrt{s}) .
$$

Hence for $R>>\sqrt{s}$ the distribution of a random vector plus a Rayleigh vector will approach that of the random vector alone. This effect may be 
observed in several cases in radio wave propagation. One of these is the field strength of VHF and UHF in cities and other built-up areas, where the total signal may be due to a direct wave (attenuated at random as it is transmitted through walls and other building materials) onto which large numbers of reflected waves are superimposed. The resulting amplitude (which is constant in time, but random when measured at different places) is then distributed as in (22). A survey conducted at various parts of the city of Prague showed that in most areas $w(\alpha)$, the distribution of the attenuated direct wave, is lognormal; the same result need not necessarily hold for cities differing in character from the above (brick or concrete houses, five to six stories high, streets relatively narrow and not forming a regular pattern). By analyzing the resulting distribution the propagation mechanism may thus be investigated (separation of reflections and attenuation).

\section{Converging Variances}

In at least two cases met in propagation theory, meteoric forward-scatter and noise due to atmospherics, the signals arrive at random intervals of time with a random amplitude which then decays exponentially. The signals are mutually independent and their phase makes them UDP vectors. Since an exponentially decaying signal never vanishes completely, there is an infinity of residual signals present at any time; but since the power at any time is finite, the infinite series of signals must converge and the denominator of (13) will not tend to infinity with $n$ (this can also be shown mathematically). Hence (13) will not be satisfied for any $j$, the Central Limit Theorem cannot be used, and the resulting amplitude distribution cannot be a pure Rayleigh distribution.

To solve the problem rigorously one therefore has to return to first principles, e.g., by finding $x$ and $y$ in (4) through their characteristic functions

$$
\begin{aligned}
\chi_{x}{ }^{(v)}=\chi_{y}{ }^{(v)} & =\prod_{j=1}^{\infty} \frac{1}{(2 \pi)^{j}} \int_{0}^{2 \pi} d \phi \int_{0}^{\infty} d E_{j} w\left(E_{j}\right) e^{i v E_{j} \cos \phi} \\
& =\prod_{j=1}^{\infty} \int_{0}^{\infty} w\left(E_{j}\right) J_{0}\left(v E_{j}\right) d E_{j}
\end{aligned}
$$

where $E_{j}$ is the amplitude of the $j$ th decaying signal, which depends on two random quantities: the time $t_{j}$ elapsed since the signal attained its peak value, and that peak value $E_{p}$ :

$$
E_{j}=E_{p} e^{-t_{j} / a}
$$

with $a$ the time constant of decay. Since the number of signals per unit time is Poisson-distributed (about an average $N), t_{1}$ has an exponential distribution

$$
w_{1}\left(t_{1}\right)=N e^{-N t_{1}}
$$

and the distribution of $t_{j}$ is given by a $j$-fold convolution of (30), which leads to

$$
w_{j}\left(t_{j}\right)=\frac{N^{j} t_{j}^{j-1}}{(j-1) !} e^{-N t_{j}} .
$$

The probability density of $B=\exp \left(-t_{j} / a\right)$ is then found from (31) by a simple transformation:

$$
w_{j}\left(B_{j}\right)=\frac{N^{j} a^{j}\left(\ln B_{j}\right)^{j-1}}{(j-1) ! B_{j}^{a N+1}} \quad(1<B<\infty) .
$$

If the density of $E_{p}$ is $\lambda\left(E_{p}\right)$, then the distribution of $E_{j}$ is found from (30) as that of a product of two random variables:

$$
w\left(E_{j}\right)=\int_{1}^{\infty} E_{j} \lambda\left(B_{j} E_{j}\right) W_{j}\left(B_{j}\right) d B_{j} .
$$

Substituting (33) in (28) will in general lead to great computational difficulties, which may be overcome by the following approximation.

From (33) we find

$$
\left\langle E_{j}^{2}\right\rangle=\int_{0}^{\infty} E_{j}^{2} w\left(E_{j}\right) d E_{j}
$$

and

$$
\left\langle E^{2}\right\rangle=\sum_{j=1}^{\infty}\left\langle E_{j}^{2}\right\rangle
$$

This series will converge and hence violate (13); however, if the series converges quickly $(N a<<1)$, then (21) will still hold for $j \neq 1$; if this term is excluded, the rest of the series may thus be approximated by a Rayleigh vector. Physically this means that if the time constant of decay is sufficiently short for the signal to decay to a low value before the next signal arrives (average interval is $1 / N$ ), then the total signal at any time will be dominated by the last signal (or possibly the last few signals), whilst the remnants of all previous signals will combine to form a low-power Rayleigh vector.

Thus under these circumstances this case may be reduced approximately to the one in section 4; the required distribution is thus given by (22) with $w(\alpha)=w\left(E_{1}\right)$ given by (33) and $s=\left\langle E^{2}\right\rangle-\left\langle E_{1}^{2}\right\rangle$ found from (34) and (35).

The distribution $\lambda\left(E_{p}\right)$ is given by the physical nature of the problem. For atmospherics, $\lambda\left(E_{p}\right)$ may be shown to be lognormal [Beckmann, 1964]; in spite of the several approximations involved, the agreement of the distribution as derived above and the measured distribution is very good as shown by figure 1. An analysis of the distribution permits the effect of propagation conditions to be separated from that of lightning activity in the total random atmospheric noise.

\section{Small Number of Independent Components}

If the number $n$ of independent interfering waves is small (e.g., the total radar signal returned from a small number of independent targets in the same 


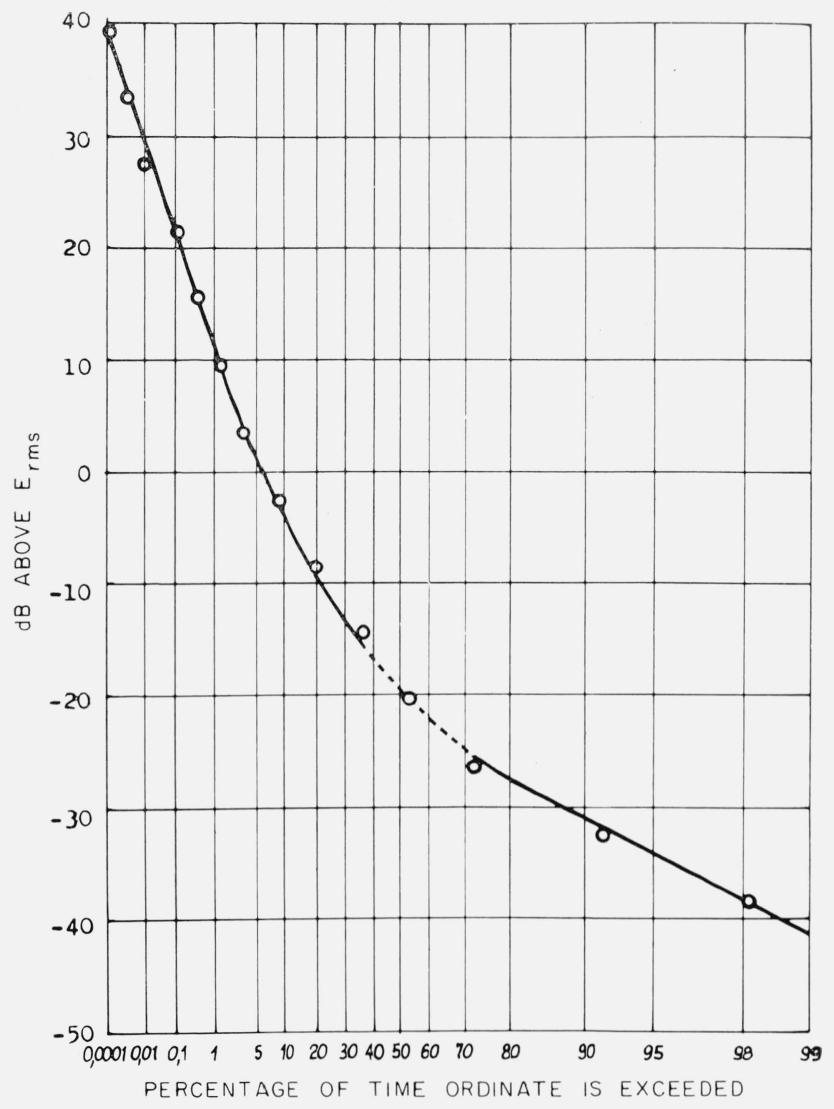

Figure 1.-Amplitude-probability distribution of atmospheric radio noise.

Circles: values measured by Crichlow et al. [1960], on $13.3 \mathrm{kc} / \mathrm{s}$ at Boulder, Colo., October 6, 1958. Full Curve: distribution computed as in (33) through (35) for $\sigma=1.67, N c=0.01$. Broken curve interpolated. (Cf. Beckmann [1964].)

area), the Central Limit Theorem cannot be applied and little can be said about the distributions of $x$ and $y$ in (4) beyond the statement that they have to be determined from the $w_{j}\left(E_{j}\right)$ by convolutions or characteristic functions. However, we may ask the opposite question: how large must $n$ be in (1) for the Rayleigh distribution to be a good approximation for $p(E)$ in engineering practice? The answer will obviously depend on the distributions $w_{j}\left(E_{j}\right)$, which we here assume all identical and equal to $w\left(E_{j}\right)$. In that case one may derive the formula [Watson, 1944; Levin, 1960]

$$
p(E)=E \int_{0}^{\infty}\left(\int_{0}^{\infty} w\left(E_{j}\right) J_{0}\left(u E_{j}\right) d E_{j}\right)^{n} u J_{0}(E u) d u \text {. }
$$

Expanding $J_{0}\left(E_{j} u\right)$ in a series and integrating term by term, rearranging in ascending powers of $(1 / n)$ and using the $2 \mathrm{~d}$ and 4 th initial moments of $w\left(E_{j}\right)$, i.e.,

$$
\begin{gathered}
m_{2}=\left\langle E_{j}^{2}\right\rangle=\int_{0}^{\infty} E_{j}^{2} w\left(E_{j}\right) d E_{j} ; \\
m_{4}=\left\langle E_{j}^{4}\right\rangle=\int_{0}^{\infty} E_{j}^{4} w\left(E_{j}\right) d E_{j}
\end{gathered}
$$

we find an expression which on integration over $E$ yields [Levin, 1960, pp. 184-187],

$$
P\left(\frac{E}{E_{\mathrm{RMS}}}>R\right)=e^{-R^{2}}\left[1+\frac{1}{4 n}\left(\frac{m_{4}}{m_{2}^{2}}-2\right) R^{4}+\ldots\right] .
$$

For $n \rightarrow \infty$ this leaves a pure Rayleigh distribution; for finite $n$, the Rayleigh distribution will be a good approximation if the second term in the square bracket will be small compared with unity; i.e., the required criterion is

$$
n>>\frac{R^{4}}{4}|\beta-2|
$$

where $\beta=m_{4} / m_{2}^{2}=\left\langle E_{j}^{4}\right\rangle /\left\langle E_{j}^{2}\right\rangle^{2}$.

\section{Random Number of Terms}

In most cases met in wave propagation through random media the number of interfering waves $n$ is not constant; the number of scatterers such as turbulence cells in the atmosphere changes from moment to moment; the number of effective reflectors in cities or irregular terrain changes from location to location, etc. Thus $n$ itself will be random; if its distribution (discrete for integers only) is $P(n)$, then the distribution of $x$ and $y$ in (4) will be

$$
p_{x}(x)=\sum_{n=0}^{\infty} P(n) \sum_{j=1}^{n} E_{j} \cos \phi_{j} .
$$

Now if $P(n)$ assumes appreciable values only for large $n$, the terms of the $n$-sum will be normal, hence $x$ will be normal and $E$ will be Rayleigh-distributed. A more detailed investigation [Beckmann, 1962b] shows that the distribution of $E$ will closely approach a Rayleigh distribution as the condition

$$
\frac{\left\langle n^{2}\right\rangle}{\langle n\rangle^{2}} \rightarrow 1
$$

is more nearly satisfied; it is also shown that for a given distribution $P(n)$ the deviation of $P(E)$ from a Rayleigh distribution will always be greater for large $E$ than for small $E$.

As a rule $n$ is Poisson-distributed about its mean value $\langle n\rangle$, i.e.,

$$
P(n)=\frac{\langle n\rangle^{n}}{n !} e^{\langle-n\rangle}
$$

From (42) we find $\left\langle n^{2}\right\rangle=\langle n\rangle+\langle n\rangle^{2}$; hence

$$
\frac{\left\langle n^{2}\right\rangle}{\langle n\rangle^{2}}=\frac{1}{\langle n\rangle}+1
$$

which will approach unity as required by (41) for $\langle n\rangle>\rangle 1$. Thus if $P(n)$ is given by (42), a large mean value is sufficient to make the Rayleigh distribution a good approximation for $p(E)$. It should 
be noted that for large $\langle n\rangle,(42)$ is equivalent to a normal distribution with $D(n)=\langle n\rangle$ [Levin, 1960].

For a general distribution $P(n)$, however, a large mean value $\langle n\rangle$ is not sufficient to guarantee that $p(E)$ in (1) and (4) will tend to the Rayleigh distribution, but the criterion (41) must be satisfied.

\section{Distribution of the Resulting Phase}

The distribution of $\theta$ in (1) is found from the second relation in (5). For a sum of UDP vectors, the resulting phase will of course again be uniformly distributed over an interval of length $2 \pi$; for nonuniform phase distributions of the vector terms we introduce the quantities

$$
\rho=\frac{r}{\sqrt{s_{1}+s_{2}}} ; B=\frac{\alpha}{\sqrt{s_{1}+s_{2}}} ; K=\sqrt{\frac{s_{2}}{s_{1}}}
$$

and then obtain from (5)

$$
p_{\theta}(\theta)=\frac{K e^{-\frac{1}{2} B^{2}\left(1+K^{2}\right)}}{2 \pi\left(K^{2} \cos ^{2} \theta+\sin ^{2} \theta\right)}\left[1+G \sqrt{\pi} e^{G^{2}}(1+\operatorname{erf} G)\right]
$$

where $^{3}$

$$
G=B K \cos \theta \sqrt{\frac{1+K^{2}}{2\left(K^{2} \cos ^{2} \theta+\sin ^{2} \theta\right)}} .
$$

\section{Reference}

Beckmann, P. (1962a), Statistical distribution of the amplitude and phase of a multiply scattered field, J. Res. NBS 66D (Radio Prop.), No. 3, 231-240.

Beckmann, P. (1962b), Deviations from the Rayleigh distribution for a small and for a random number of interfering waves, URE-ČSAV Inst. Rept. No. 25.

Beckmann, P. (1964), The amplitude-probability distribution of atmospheric noise, Radio Sci. J. Res. NBS/USNC/URSI 68D, No. 6, 723-735.

${ }^{3}$ Note the factor $\cos \theta$ in (46), which unfortunately dropped out from the corresponding equation on p. 236 of Beckmann [1962a].
Crichlow, W. Q., C. J. Roubique, A. D. Spaulding, and W M. Beery (Jan.-Feb. 1960), Determination of the amplitude-probability distribution of atmospheric radio noise from statistical moments, J. Res. NBS 64D (Radio Prop.), No. 1, 49-56.

Gnedenko, B. V., and A. N. Kolmogorov (1954), Limit distributions for sums of independent random variables (Addison-Wesley Publishing Co., Cambridge, Mass.).

Hoyt, R. S. (1947), Probability functions for the modulus and angle of the normal complex variate, Bell System Tech. J. 26, 318-359.

Levin, B. R. (1960), The theory of random processes and its application to radio engineering, $2 \mathrm{~d}$ ed. (in Russian) Sovyetskoye Radio, Moscow.

Nagakami, M. (1960), The $m$-distribution-a general formula of intensity distribution of rapid fading, Statistical Methods in Radio Wave Propagation, ed. W. C. Hoffman (Pergamon Press, Oxford).

Norton, K. A., L. E. Vogler, W. V. Mansfield, and P. J. Short (1955), The probability distribution of the amplitude of a constant vector plus a Rayleigh-distributed vector, Proc. IRE 53, 1354-1361.

Ravleigh, Lord (1896), The Theory of Sound, sec. 42a, 2d ed. (Macmillan, London), (Reprinted Dover, 1945).

Rice, S. O. (1944), Mathematical analysis of random noise, Bell System Tech. J. 23, 282-332.

Rice, S. O. (1945), Mathematical analysis of random noise, Bell System Tech. J. 24, 46-156.

Watson, G. N. (1944), A Treatise on the Theory of Bessel Functions (University Press, Cambridge).

Zuhrt, H. (1957), Die Summenhäufigkeitskurven der exzentrischen Rayleigh-Verteilung und ihre Anwendung auf Ausbreitungsmessungen, A. E. Ü. 11, 478-484.

\section{Additional Related References}

Furutsu, K., and T. Ishida (July 1961), On the theory of amplitude distribution of impulsive random noise, J. Appl. Phys. 32, No. \%, 1206-1221.

Siddiqui, M. M. (Mar.-Apr. 1962), Some problems connected with Rayleigh distribution, J. Res. NBS 66D (Radio Prop.), No. 2, 167-174.

Wheelon, Albert D. (Sept.-Oct. 1960), Amplitude distribution for radio signals reflected by meteor trails, J. Res. NBS 64D (Radio Prop.), No. 5, 449-454.

Wheelon, Albert D. (May-June 1962), Amplitude distribution for radio signals reflected by meteor trails, II, J. Res. NBS 661 (Radio Prop.), No. 3, 241-247.

(Paper 68D9-392) 